\title{
Reconstruction of hv-convex sets by their coordinate X-ray functions
}

\author{
Ábris Nagy* Csaba Vincze ${ }^{\dagger}$
}

February 6, 2014

\begin{abstract}
R. J. Gardner and M. Kiderlen [17] presented an algorithm for reconstructing convex bodies from noisy X-ray measurements with a full proof of convergence in 2007. We would like to present some new steps into the direction of reconstructing not necessarily convex bodies by the help of the continuity properties of so-called generalized conic functions. Such a function measures the average taxicab distance of the points from a given compact set $K \subset \mathbb{R}^{N}$ by integration. The basic result [28] is that the generalized conic function associated to a compact planar set determines the coordinate X-rays and vice versa. Cs. Vincze and Á Nagy [29] proved continuity properties of the mapping which sends connected compact hv-convex sets having the same axis parallel bounding box to the associated generalized conic functions. We use these results to present an algorithm for the reconstruction of compact connected hv-convex planar bodies given by their coordinate X-rays. The basic method is varied with the quota system scheme. Greedy and anti-greedy versions are also presented with examples.
\end{abstract}

Key words: parallel X-ray, generalized conic, geometric tomography, linear integer programming

\section{Introduction}

'Tomography refers to the cross-sectional imaging of an object from either transmission or reflection data collected by illuminating the object from many different directions' [25]. From the mathematical point of view this means the reconstruction of a density distribution by its integrals along

\footnotetext{
*Institute of Mathematics, MTA-DE Research Group "Equations Functions and Curves", Hungarian Academy of Sciences and University of Debrecen, P. O. Box 12, 4010 Debrecen, Hungary, abris.nagy@science.unideb.hu

${ }^{\dagger}$ Institute of Mathematics, University of Debrecen, P. O. Box 12, 4010 Debrecen, Hungary, csvincze@science.unideb.hu
} 
straight lines. Basic methods to carry out this reconstruction are based on Fourier transformation, while algebraic reconstruction techniques are implemented in the case of pixel-based objects (i.e. the density distribution is assumed to be constant in each cell of a square grid). A detailed overview of these techniques is presented in [23] and [25]. 'Geometric tomography is the area of mathematics dealing with the retrieval of information about a geometric object from data about its intersections, or projections, or both' [14]. The word geometric is used here because we assume that the object is homogeneous in contrast to tomography. In other words we are only interested in the shape of the object. In the homogeneous case the density distribution becomes to be the characteristic function of the object. Parallel X-rays are functions that measure the intersection of a given set with hyperplanes parallel to a fixed subspace of codimension 1 of $\mathbb{R}^{N}$. The reconstruction problem concerning parallel X-rays is to reconstruct a compact subset of $\mathbb{R}^{N}$ if the parallel X-rays of this set into some directions are given. In many cases it is enough to construct a sequence of sets that converges to a compact set with respect to the Hausdorff distance, and has the given X-rays.

First we can use geometric variants of the reconstruction tools of tomography, such as binary or discrete algebraic reconstruction techniques (see [5] and [22]). Other iterative approaches have been employed also, such as gradient descent algorithm or maximum-likelihood expectation-maximization. Recent different techniques are, for instance, the model-based iterative reconstruction (see [31]), the combination with compressed sensing (see [32]), or the use of adaptive scanning patterns during the acquisition of the projections (see [21]). These techniques reduce the number of projections needed, but additional knowledge on the object is needed to obtain a unique solution. For example, assuming convexity of the object leads us to a much better situation where a suitable finite number of directions is enough for all convex bodies to be uniquely determined by their X-rays in these directions. Gardner and McMullen showed in [18] that there are certain sets of four directions, such that every convex body in $\mathbb{R}^{2}$ is determined by its X-ray functions in these directions, but there is no set of three directions with the same property. Later Gardner and Gritzmann showed in [15] that suitable sets of four directions are those whose set of slopes, in increasing order, have a rational cross-ratio not equal to $3 / 2,4 / 3,2,3$ or 4 . This result is related to the geometric structure of convex-shaped switching components, known in the literature as $U$-polygons, where $U$ is the set of employed directions (see [10], [12] and [24]). These results for convex planar bodies can be used to verify similar statements in higher dimensions with the help of a slice-by-slice technique.

In the contest of point X-rays, uniqueness of reconstruction is guaranteed for convex bodies of $\mathbb{R}^{2}$ by means of X-rays taken in a set of four sources, no three collinear (see [30]). An analogous result doesn't hold in discrete tomography where finding the minimum number of sources, in general posi- 
tion, requested for uniqueness is still an open problem (see [11]). However, the problem of unique determination under a finite number of directions is largely investigated in discrete tomography, even without the convexity assumption but with some kind of a priori knowledge (for details see [9], [13], [16] and [19]). An algorithm for reconstructing convex lattice set is presented in [6]. Reconstruction of continuous objects (i.e. no lattice sets) is much harder, even if convexity is assumed. Gardner and Kiderlen present an algorithm in [17] for reconstructing convex bodies of $\mathbb{R}^{2}$ from noisy $\mathrm{X}$ ray measurements with a full proof of the convergence. The main feature of this algorithm is that it works in the case when the data is noisy and uses only four directions. Earlier reconstruction algorithms are presented in [8] and [26], but they suffer from several problems (see the introduction part of [17]).

The purpose of this paper is to give an algorithm for the reconstruction of planar bodies by their X-rays that works not only for the convex case and uses a finite number of fixed directions. The situation is much harder in this case since polygons in $\mathbb{R}^{2}$ that are star-shaped at the origin are not determined by X-rays in any finite set of directions. This shows that we need some additional assumption that is a little weaker than convexity. This assumption is hv-convexity. The reconstruction of hv-convex sets is has been extensively considered in discrete tomography ([2], [3] and [4]), but now we would like to focus on continuous objects of $\mathbb{R}^{2}$. Brunetti and Daurat approximate Q-convex bodies with Q-convex lattice sets in [7] and suggest to use an algorithm applied in discrete tomography, but they do not prove that their algorithm converges. The convergence of our reconstruction algorithm is shown in Section 5, after the algorithm is presented in Section 4. Preliminaries and basic theorems from our main reference works ([28] and [29]) are the subject of Section 2 and 3, while in Section 6 we show how to apply linear integer programming to execute the main step of the algorithm. After that greedy and anti-greedy versions are also presented varied with a quota system scheme in Section 7 and 8.

\section{Preliminaries}

In what follows we collect the basic facts of the theory. Let $\mathbb{R}^{N}$ be the Ndimensional real coordinate space with the standard basis $\underline{e}_{1}, \ldots, \underline{e}_{N}$. Vectors of the form $\underline{x}=\left(x_{1}, x_{2}, \ldots, x_{N}\right)$ denote elements of $\mathbb{R}^{N}$. For $p \in \mathbb{Z}, p \geq 1$, and $\underline{x} \in \mathbb{R}^{N}$, let $\|\underline{x}\|_{p}$ be the $p$-norm of $\underline{x}$ and consider the distance function $d_{p}$ induced by the $p$-norm:

$$
\|\underline{x}\|_{p}=\sqrt[p]{\sum_{i=1}^{N}\left|x_{i}\right|^{p}}, \quad d_{p}(\underline{x}, \underline{y})=\|\underline{x}-\underline{y}\|_{p} .
$$


Throughout this paper $\mu_{N}$ will denote the $N$-dimensional Lebesgue measure $(N \in \mathbb{N}, N>0)$, while $D_{i}=\frac{\partial}{\partial x_{i}}$ is the differential operator of partial differentiation with respect to the i-th coordinate $(i=1, \ldots, N)$. The Hausdorff distance between (compact) sets $L$ and $K$ will be denoted by $H(L, K)$, as usual. A compact set $K \subset \mathbb{R}^{N}$ is called a body if $K$ has a nonempty interior. For any $c \in \mathbb{R}$ let us define the sets

$$
\begin{array}{ll}
K<_{i} c:=\left\{\underline{x} \in K \mid x_{i}<c\right\} & (i=1, \ldots, N), \\
c<_{i} K:=\left\{\underline{x} \in K \mid c<x_{i}\right\} & (i=1, \ldots, N), \\
c={ }_{i} K:=\left\{\underline{x} \in K \mid c=x_{i}\right\} & (i=1, \ldots, N) .
\end{array}
$$

Definition 1 A planar set $K \subset \mathbb{R}^{2}$ is said to be hv-convex if the sections $c={ }_{i} K(i=1,2)$ are convex sets for all $c \in \mathbb{R}$.

Definition 2 The $X$-ray functions of a compact set $K \subset \mathbb{R}^{N}$ into the coordinate directions are

$$
X_{i} K: \mathbb{R} \rightarrow \mathbb{R}, \quad t \mapsto X_{i} K(t):=\mu_{N-1}\left(t={ }_{i} K\right) \quad(i=1, \ldots, N) .
$$

For a general definition of X-ray functions and general observations on these functions see chapters 1 and 2 in Gardner's book [14].

Definition 3 Let $\mathcal{E}$ be a class of bounded measurable sets of $\mathbb{R}^{N}$ and $S$ a fixed set of directions. We say that $E \in \mathcal{E}$ is determined by the $X$-rays parallel to the directions in $S$ if whenever $E^{\prime} \in \mathcal{E}$ and the $X$-rays of $E$ and $E^{\prime}$ coincide almost everywhere for all directions in $S$, we have $\mu_{N}\left(E \triangle E^{\prime}\right)=0$, i.e. the symmetric difference of $E$ and $E^{\prime}$ is a set of measure zero.

Now we collect some results on wich sets are determined by their coordinate X-rays, shown in [28] for $N=2$. Since the proofs are coordinatewise, they immediately generalize to higher dimensions.

Definition 4 The generalized $p$-conic function $f_{K}^{p}$ associated to a compact set $K$ is

$$
f_{K}^{p}: \mathbb{R}^{N} \rightarrow \mathbb{R}, \quad \underline{x} \mapsto f_{K}^{p}(\underline{x}):=\int_{K} d_{p}(\underline{x}, \underline{y}) d \underline{y} .
$$

The level sets $C_{K}^{p}=\left\{\underline{x} \in \mathbb{R}^{N} \mid f_{K}^{p}(\underline{x}) \leq c\right\}$ are called generalized $p$-conics.

For the sake of simplicity generalized 1-conics will be simply called generalized conics, while generalized 1-conic functions will be called generalized conic function: $f_{K}:=f_{K}^{1}$ for every compact set $K \subset \mathbb{R}^{N}$.

Lemma 1 (Vincze, Nagy [28]) For any compact body $K \subset \mathbb{R}^{N}$

$$
D_{i} f_{K}(\underline{x})=\mu_{N}\left(K<_{i} x_{i}\right)-\mu_{N}\left(x_{i}<_{i} K\right) \quad(i=1, \ldots, N) .
$$


Theorem 1 (Vincze, Nagy [28]) For compact bodies $K$ and $K^{*}$ in $\mathbb{R}^{N}$ $f_{K}=f_{K^{*}}$ if and only if $X_{i} K=X_{i} K^{*}$ for all $i \in\{1, \ldots, N\}$ almost everywhere.

The coordinate X-rays can be expressed explicitly by the generalized conic function and vice versa. In terms of the higher dimensional setting

$$
\begin{aligned}
D_{i} D_{i} f_{K}(\underline{x}) & ={ }_{\text {a.e. }} 2 X_{i} K\left(x_{i}\right) \quad(i=1, \ldots, N), \\
f_{K}(\underline{x}) & =\sum_{i=1}^{N} \int_{-\infty}^{\infty}\left|x_{i}-t\right| X_{i} K(t) d t .
\end{aligned}
$$

Theorem 2 (Vincze, Nagy [28]) Let $C$ be a generalized $p$-conic and suppose that $C^{*}$ is a bounded measurable set with $\mu_{N}(C)=\mu_{N}\left(C^{*}\right)$. If the generalized $p$-conic functions associated to $C$ and $C^{*}$ coincide then $\mu_{N}\left(C \triangle C^{*}\right)=$ 0 .

Corollary 1 Generalized conics are determined by their X-rays in the coordinate directions among compact bodies of $\mathbb{R}^{N}$.

Example. Balls are determined by their X-rays in the coordinate directions among compact bodies because they are level sets of the generalized conic function associated to the circumscribed cube: in case of the cube $B:=$ $[a, b]^{N}$, where $a, b \in \mathbb{R}, a<b$ we have that

$$
f_{B}(\underline{x})=(b-a)^{N-1} \sum_{i=1}^{N}\left(\left(x_{i}-\frac{a+b}{2}\right)^{2}+\frac{(b-a)^{2}}{4}\right)
$$

for any interior point $\underline{x}$.

We also mention the following result due to R. J. Gardner on convex planar bodies determined by two X-rays.

Theorem 3 (Gardner [14]) Given any two nonparallel directions, the set of convex planar bodies determined by their $X$-rays in these directions is of second category in the metric space of convex planar bodies equipped with the Hausdorff metric.

\section{Continuity properties}

In what follows we use the main results of our reference work [29] to present an algorithm for the reconstruction of a connected hv-convex planar body $K$ given by the coordinate $\mathrm{X}$-rays. 
Definition 5 Consider a non-empty compact planar set $K$ and let $B_{K}$ be the intersection of all rectangles having sides parallel to the coordinate directions and containing $K$. Then $B_{K}$ is called the axis parallel bounding box of $K$.

If $K \subset \mathbb{R}^{2}$ is a connected body and $K=\operatorname{cl}(\operatorname{int}(K))(K$ is the closure of its interior) then

$$
B_{K}=\operatorname{supp}\left(X_{1} K\right) \times \operatorname{supp}\left(X_{2} K\right) \text {. }
$$

Let $\mathcal{L}_{B}^{h v}$ denote the collection of non-empty compact hv-convex sets having the same axis parallel bounding box $B:=[a, b] \times[c, d]$.

Theorem 4 (Vincze,Nagy [29]) The mapping $\Phi: L \in \mathcal{L}_{B}^{h v} \mapsto f_{L}$ is continuous between $\mathcal{L}_{B}^{h v}$ equipped with the Hausdorff metric and the function space equipped with the $L^{1}$-norm

$$
\left|f_{L}\right|_{1}:=\int_{B}\left|f_{L}\left(x_{1}, x_{2}\right)\right| d x_{1} d x_{2} .
$$

Theorem 5 (Vincze,Nagy [29]) The mapping $\Phi: L \in \mathcal{L}_{B}^{h v} \mapsto f_{L}$ is continuous between $\mathcal{L}_{B}^{h v}$ equipped with the Hausdorff metric and the function space equipped with the supremum norm

$$
\left|f_{L}\right|_{\infty}:=\sup _{\left(x_{1}, x_{2}\right) \in B}\left|f_{L}\left(x_{1}, x_{2}\right)\right| .
$$

These continuity properties are crucial for the reconstruction because they imply that the inverse $\Phi^{-1}$ is upper semi-continuous as a set-valued mapping. The practical formulation can be found in the following corollary.

Corollary 2 (Vincze,Nagy [29]) Let $L_{n}$ be a sequence of non-empty compact connected hv-convex sets having the same axis parallel bounding box $B$. If $f_{L_{n}} \rightarrow f_{K}$ with respect to the $L^{1}$-norm or the supremum norm then any convergent subsequence of $L_{n}$ tends to a set $K^{*} \in \mathcal{L}_{B}^{h v}$ satisfying $f_{K}=f_{K^{*}}$, i.e. $K^{*}$ and $K$ have the same coordinate $X$-rays almost everywhere. In particular if $K$ is uniquely determined in $\mathcal{L}_{B}^{h v}$ by the coordinate $X$-rays then $\mu_{2}\left(K \triangle K^{*}\right)=0$, i.e. the symmetric difference of $K$ and $K^{*}$ is a set of measure zero.

Proof. We discuss only the case of the $L^{1}$-norm (the case of the supremum norm is similar). Suppose that $K^{*}$ is the limit of a convergent subsequence $L_{n_{k}}$. Then

$$
\int_{B}\left|f_{K^{*}}\left(x_{1}, x_{2}\right)-f_{K}\left(x_{1}, x_{2}\right)\right| d x_{1} d x_{2} \leq
$$


$\int_{B}\left|f_{K^{*}}\left(x_{1}, x_{2}\right)-f_{L_{n_{k}}}\left(x_{1}, x_{2}\right)\right| d x_{1} d x_{2}+\int_{B}\left|f_{L_{n_{k}}}\left(x_{1}, x_{2}\right)-f_{K}\left(x_{1}, x_{2}\right)\right| d x_{1} d x_{2}$,

where the first term tends to zero in view of Theorem 4 . So does the second term because of the condition $f_{L_{n}} \rightarrow f_{K}$ with respect to the $L^{1}$-norm. Taking the limit as $k \rightarrow \infty$

$$
\int_{B}\left|f_{K^{*}}\left(x_{1}, x_{2}\right)-f_{K}\left(x_{1}, x_{2}\right)\right| d x_{1} d x_{2}=0
$$

which means that $f_{K}\left(x_{1}, x_{2}\right)=f_{K^{*}}\left(x_{1}, x_{2}\right)$ for any $\left(x_{1}, x_{2}\right) \in B$ because of the continuity of the generalized conic functions. Therefore $K$ and $K^{*}$ have the same coordinate X-rays almost everywhere.

As a sequence-free version of Corollary 2 we can formulate the following theorem of approximation. The norm of the functions can be considered alternatively both the $L^{1}$-norm or the supremum norm (over B).

Theorem 6 Suppose that $K \subset \mathbb{R}^{2}$ is a non-empty compact connected and $h v$-convex set with axis parallel bounding box $B$. For any $\varepsilon>0$ there exists $\delta>0$ such that for any $L \in \mathcal{L}_{B}^{h v}$

$$
\left|f_{L}-f_{K}\right|<\delta
$$

implies that $H\left(L, K^{*}\right)<\varepsilon$ for some $K^{*} \in \mathcal{L}_{B}^{h v}$, where $K^{*}$ has the same coordinate $X$-rays as $K$ almost everywhere. In particular if $K$ is uniquely determined in $\mathcal{L}_{B}^{h v}$ by the coordinate $X$-rays then $\mu_{2}\left(K \triangle K^{*}\right)=0$.

This theorem says that if $f_{L} \approx f_{K}$ with respect to the $L^{1}$-norm or the supremum norm then $L$ approximates at least one of the sets in $\mathcal{L}_{B}^{h v}$ with the same coordinate $\mathrm{X}$-rays as $K$ almost everywhere.

\section{An algorithm for the reconstruction of hv-convex sets by their coordinate X-ray functions}

In geometric tomography there are satisfying results on how to choose directions in the plane to ensure some classes of sets to be determined by X-rays in these directions (see Chapter 1 in [14]). However these results do not provide any algorithm for reconstruction. In fact there are only a few attempts to provide such an algorithm with a proof of convergence. One recent paper on this topic is [17], where an algorithm is provided that takes as input $n$ equally spaced noisy X-ray measurements of an unknown planar convex body $K$ in each of some fixed directions, and produces a convex polygon $P_{n}$ such that $P_{n}$ almost surely converges to $K$ with respect to the Hausdorff metric as $n \rightarrow \infty$. The noise is modeled in the traditional way by adding 
independent $N\left(0, \sigma^{2}\right)$ random variables. The directions are chosen such that any convex planar body is determined by its X-rays in these directions.

The main feature in [17] is the complete proof of the convergence of their algorithm that applies also for the case when the data are noisy. We also need to mention that the algorithm takes the measurements of the X-ray functions only in a finite set of points, however the number of these points must increase if we want a better approximation. On the other hand the algorithm is purely theoretical and seems to be hard to implement. The main step consists in solving a constrained least-squares problem with $8 n$ continuous variables and with an objective function that is heavily nonlinear (e.g. it is not partially differentiable). In a very recent paper [1] an implementation of this algorithm in Matlab is compared with other geometric reconstruction techniques.

Now we would like to present an algorithm that works not only for convex bodies and can be implemented easily. The convergence of this algorithm is shown in the next section.

Input: $n \in \mathbb{N}$ and $X_{1} K, X_{2} K$, the coordinate X-rays of a connected hvconvex body $K \subset \mathbb{R}^{2}$ with $K=\operatorname{cl}(\operatorname{int}(K))$ and having axis parallel bounding box $B_{K}$.

The process involves the following basic theoretical steps:

STEP 1: Compute the box as the product $B_{K}=[a, b] \times[c, d]$ and the function $f_{K}$ associated to $K$ by (2) and (1).

STEP 2: Let $t_{i} \in[a, b]$ and $s_{i} \in[c, d],(i=0, \ldots, n)$ be equally spaced points with $t_{0}=a, t_{n}=b$ and $s_{0}=d, s_{n}=c$ :

$$
t_{i}=a+i \frac{b-a}{n}, \quad s_{i}=d-i \frac{d-c}{n} \quad(i=0, \ldots, n) .
$$

STEP 3: $B_{i j}^{n}=\left[t_{i-1}, t_{i}\right] \times\left[s_{j}, s_{j-1}\right] \quad(i, j=1, \ldots, n)$.

STEP 4: The control grid $G_{K}^{n}$ of $K$ is defined as the set of the centers of the subrectangles $B_{i j}^{n}$ :

$$
G_{K}^{n}:=\left\{\underline{y}_{i j} \in B_{K} \mid i, j=1, \ldots, n\right\},
$$

where

$$
\underline{y}_{i j}=\left(\frac{t_{i-1}+t_{i}}{2}, \frac{s_{j-1}+s_{j}}{2}\right)=\left(a+\frac{2 i-1}{2 n}(b-a), d-\frac{2 j-1}{2 n}(d-c)\right) .
$$

STEP 5: Let $\mathcal{H}$ be the collection of all sets $L \in \mathcal{L}_{B_{K}}^{h v}$ such that $L$ is the union of some subrectangles $B_{i j}^{n}$ presented in the third step and $f_{L}\left(\underline{y}_{i j}\right) \geq f_{K}\left(\underline{y}_{i j}\right)$ for all $\underline{y}_{i j} \in G_{K}^{n}$. 
STEP 6: Choose $L_{n}$ from $\mathcal{H}$ that minimizes

$$
\sum_{i, j=1}^{n} \frac{f_{L_{n}}\left(\underline{y}_{i j}\right)-f_{K}\left(\underline{y}_{i j}\right)}{n^{2}} .
$$

Alternatively we have

STEP 6*: Choose $L_{n}$ from $\mathcal{H}$ that minimizes

$$
\max _{\underline{y}_{i j} \in G_{K}^{n}}\left(f_{L_{n}}\left(\underline{y}_{i j}\right)-f_{K}\left(\underline{y}_{i j}\right)\right) \text {. }
$$

Output: $L_{n}$

\section{The proof of the convergence}

In what follows we prove that any convergent subsequence of the output sequence $L_{n}$ converges to a compact connected hv-convex planar set $K^{*}$ such that $f_{K}=f_{K^{*}}$ which means by Theorem 1 that $K$ and $K^{*}$ have the same coordinate X-rays almost everywhere. Note that if $K=\operatorname{cl}(\operatorname{int}(K))$ then $\mu_{2}\left(K \triangle K^{*}\right)=0$ implies $K=K^{*}$. This gives that if $K$ is uniquely determined by the coordinate X-rays then $L_{n} \rightarrow K$ with respect to the Hausdorff metric. Let us start with a relatively simple but important observation.

Lemma 2 For any compact set $K \subset \mathbb{R}^{2}$

$$
\left|f_{K}(\underline{y})-f_{K}(\underline{x})\right| \leq\|\underline{y}-\underline{x}\|_{1} \mu_{2}(K)
$$

Proof. Recall that the 1-norm is decomposable. So is $f_{K}$. If $\underline{x}=\left(x_{1}, x_{2}\right)$ and $\underline{y}=\left(y_{1}, y_{2}\right)$ then we have by the classical mean value theorem for functions with one variable that

$$
\left|f_{K}(\underline{y})-f_{K}(\underline{x})\right| \leq\left|D_{1} f_{K}\left(t^{*}, x_{2}\right)\right| \cdot\left|y_{1}-x_{1}\right|+\left|D_{2} f_{K}\left(y_{1}, s^{*}\right)\right| \cdot\left|y_{2}-x_{2}\right|
$$

for some reals $t^{*}$ and $s^{*}$ between the corresponding coordinates of the given points, respectively. Lemma 1 provides the Lebesgue measure $\mu_{2}(K)$ as the upper bound for the absolute value of derivatives with respect to both the first and the second variables.

Theorem 7 Any convergent subsequence of the output sequence $L_{n}$ converges to a compact connected hv-convex planar set $K^{*}$ such that $K^{*}$ has the coordinate $X$-rays $X_{1} K$ and $X_{2} K$ almost everywhere. Especially if $K$ is uniquely determined by the coordinate $X$-rays then $L_{n}$ tends to $K$ with respect to the Hausdorff metric. 
Remark 1 Another possible formulation of Theorem 7 is that for any $\varepsilon>0$ there exists $n \in \mathbb{N}$ such that $H\left(L_{n}, K^{*}\right)<\varepsilon$ for some non-empty compact connected hv-convex set $K^{*}$ having the same coordinate $X$-rays as $K$ almost everywhere. Especially if $K$ is uniquely determined by the coordinate $X$-rays then $H\left(L_{n}, K\right)<\varepsilon$.

Proof. First, consider the objective function in STEP 6 which is obviously related to the $L^{1}$-norm. We have that

$$
\begin{gathered}
\int_{B}\left|f_{L_{n}}(\underline{x})-f_{K}(\underline{x})\right| d \underline{x}=\sum_{i, j} \int_{B_{i j}^{n}}\left|f_{L_{n}}(\underline{x})-f_{K}(\underline{x})\right| d \underline{x}= \\
\mu_{2}(B) \sum_{i, j} \frac{\left|f_{L_{n}}\left(\underline{x}_{i j}\right)-f_{K}\left(\underline{x}_{i j}\right)\right|}{n^{2}}
\end{gathered}
$$

for some elements $\underline{x}_{i j} \in B_{i j}^{n}$, where $i$ and $j$ run from 1 to $n \in \mathbb{N}$; this follows immediately from the first mean value theorem for integration. Using the points $\underline{y}_{i j}$ of the control grid

$$
\begin{gathered}
\sum_{i, j} \frac{\left|f_{L_{n}}\left(\underline{x}_{i j}\right)-f_{K}\left(\underline{x}_{i j}\right)\right|}{n^{2}} \leq \sum_{i, j} \frac{\left|f_{L_{n}}\left(\underline{x}_{i j}\right)-f_{L_{n}}\left(\underline{y}_{i j}\right)\right|}{n^{2}}+ \\
\sum_{i, j} \frac{\left|f_{L_{n}}\left(\underline{y}_{i j}\right)-f_{K}\left(\underline{y}_{i j}\right)\right|}{n^{2}}+\sum_{i, j} \frac{\left|f_{K}\left(\underline{y}_{i j}\right)-f_{K}\left(\underline{x}_{i j}\right)\right|}{n^{2}} .
\end{gathered}
$$

Using Lemma 2

$$
\sum_{i, j} \frac{\left|f_{L_{n}}\left(\underline{x}_{i j}\right)-f_{K}\left(\underline{x}_{i j}\right)\right|}{n^{2}} \leq 2 \mu_{2}(B) \sum_{i, j} \frac{\left\|\underline{x}_{i j}-\underline{y}_{i j}\right\|_{1}}{n^{2}}+\sum_{i, j} \frac{\left|f_{L_{n}}\left(\underline{y}_{i j}\right)-f_{K}\left(\underline{y}_{i j}\right)\right|}{n^{2}} .
$$

Since $L_{n}$ minimizes the value of the second term among the elements of $\mathcal{H}$ and

$$
\left\|\underline{x}_{i j}-\underline{y}_{i j}\right\|_{1} \leq \frac{k}{4 n}<\frac{k}{2 n},
$$

where $k$ is the perimeter of the box of $K$, it follows that

$$
\sum_{i, j} \frac{\left|f_{L_{n}}\left(\underline{x}_{i j}\right)-f_{K}\left(\underline{x}_{i j}\right)\right|}{n^{2}} \leq \mu_{2}(B) \sum_{i, j} \frac{k}{n^{3}}+\sum_{i, j} \frac{\left|f_{L_{n}^{*}}\left(\underline{y}_{i j}\right)-f_{K}\left(\underline{y}_{i j}\right)\right|}{n^{2}},
$$

where

$$
L_{n}^{*}:=\bigcup_{B_{i j}^{n} \cap K \neq \emptyset} B_{i j}^{n}
$$


is the minimal covering ${ }^{1}$ of $K$. For any $\underline{y}_{i j} \in G_{K}^{n}$

$$
\left|f_{L_{n}^{*}}\left(\underline{y}_{i j}\right)-f_{K}\left(\underline{y}_{i j}\right)\right|=f_{L_{n}^{*}}\left(\underline{y}_{i j}\right)-f_{K}\left(\underline{y}_{i j}\right)=\int_{L_{n}^{*} \backslash K} d_{1}\left(\underline{y}_{i j}, \underline{x}\right) d \underline{x} \leq \frac{k}{2} \mu_{2}\left(L_{n}^{*} \backslash K\right),
$$

where the half of the perimeter is just the diameter of the box with respect to the 1 - norm. The minimal covering is obviously an outer Hausdorff approximation of $K$ which is regular in the sense that

$$
\lim _{n \rightarrow \infty} \mu_{2}\left(L_{n}^{*} \backslash K\right)=\lim _{n \rightarrow \infty} \mu_{2}\left(L_{n}^{*}\right)-\mu_{2}(K)=0 .
$$

Therefore

$$
\begin{gathered}
\sum_{i, j} \frac{\left|f_{L_{n}}\left(\underline{x}_{i j}\right)-f_{K}\left(\underline{x}_{i j}\right)\right|}{n^{2}} \leq \mu_{2}(B) \sum_{i, j} \frac{k}{n^{3}}+\sum_{i, j} \frac{\left|f_{L_{n}^{*}}\left(\underline{y}_{i j}\right)-f_{K}\left(\underline{y}_{i j}\right)\right|}{n^{2}} \leq \\
\mu_{2}(B) \sum_{i, j} \frac{k}{n^{3}}+c_{n} \sum_{i, j} \frac{k}{2 n^{2}},
\end{gathered}
$$

where $c_{n}:=\mu_{2}\left(L_{n}^{*}\right)-\mu_{2}(K)$ tends to zero ${ }^{2}$. Finally

$$
\lim _{n \rightarrow \infty} \sum_{i, j} \frac{\left|f_{L_{n}}\left(\underline{x}_{i j}\right)-f_{K}\left(\underline{x}_{i j}\right)\right|}{n^{2}} \leq \mu_{2}(B) \lim _{n \rightarrow \infty} \frac{k}{n}+\frac{k}{2} \lim _{n \rightarrow \infty} c_{n}=0
$$

and we have that

$$
\lim _{n \rightarrow \infty} \int_{B}\left|f_{L_{n}}(\underline{x})-f_{K}(\underline{x})\right| d \underline{x}=0 .
$$

This means that $f_{L_{n}}$ tends to $f_{K}$ with respect to the $L^{1}$ - norm and the statement is a direct consequence of Corollary 2 .

Second, consider the objective function in STEP $\mathbf{6}^{*}$ which is obviously related to the supremum norm. We have that

$$
\sup _{\underline{x} \in B}\left|f_{L_{n}}(\underline{x})-f_{K}(\underline{x})\right|=\left|f_{L_{n}}\left(\underline{x}^{*}\right)-f_{K}\left(\underline{x}^{*}\right)\right|
$$

\footnotetext{
${ }^{1}$ Note that $L_{n}^{*}$ belongs automatically to $\mathcal{H}$.

${ }^{2}$ To get some information on the speed of convergence we should estimate the sequence $c_{n}$. We have that

$$
c_{n}:=\mu_{2}\left(L_{n}^{*}\right)-\mu_{2}(K) \leq \mu_{2}\left(K^{r_{n}}\right)-\mu_{2}(K),
$$

where $r_{n}$ is just the (common) diameter of the subrectangles $B_{i j}^{n}$. It can be easily seen that

$$
r_{n}=\frac{d}{n}
$$

where $d$ is the diameter of the box $B_{K}$. Therefore, by Theorem 2 in [29],

$$
c_{n} \leq \mu_{2}\left(K^{r_{n}}\right)-\mu_{2}(K) \leq 2 k \frac{d}{n} .
$$
}


for some element $\underline{x}^{*} \in B$ because the generalized conic functions are convex and, consequently, continuous functions on the coordinate plane. Let $\underline{y}^{*}$ be the center of the subrectangle containing $\underline{x}^{*}$. Then

$$
\begin{gathered}
\left|f_{L_{n}}\left(\underline{x}^{*}\right)-f_{K}\left(\underline{x}^{*}\right)\right| \leq \\
\left|f_{L_{n}}\left(\underline{x}^{*}\right)-f_{L_{n}}\left(\underline{y}^{*}\right)\right|+\left|f_{L_{n}}\left(\underline{y}^{*}\right)-f_{K}\left(\underline{y}^{*}\right)\right|+\left|f_{K}\left(\underline{y}^{*}\right)-f_{K}\left(\underline{x}^{*}\right)\right| \leq \\
2 \mu_{2}(B)\left\|\underline{x}^{*}-\underline{y}^{*}\right\|_{1}+\left|f_{L_{n}}\left(\underline{y}^{*}\right)-f_{K}\left(\underline{y}^{*}\right)\right|
\end{gathered}
$$

because of Lemma 2 . Keeping in mind that $\underline{y}^{*}$ belongs to the control grid it follows that

$$
\left|f_{L_{n}}\left(\underline{x}^{*}\right)-f_{K}\left(\underline{x}^{*}\right)\right| \leq \mu_{2}(B) \frac{k}{n}+\sup _{i, j}\left|f_{L_{n}}\left(\underline{y}_{i j}\right)-f_{K}\left(\underline{y}_{i j}\right)\right| .
$$

By the help of the minimal covering $L_{n}^{*}$ the proof can be finished as in the first case:

$$
\begin{gathered}
\left|f_{L_{n}}\left(\underline{x}^{*}\right)-f_{K}\left(\underline{x}^{*}\right)\right| \leq \mu_{2}(B) \frac{k}{n}+\sup _{i j}\left|f_{L_{n}^{*}}\left(\underline{y}_{i j}\right)-f_{K}\left(\underline{y}_{i j}\right)\right| \leq \\
\mu_{2}(B) \frac{k}{n}+\frac{k}{2} \mu_{2}\left(L_{n}^{*} \backslash K\right)=\mu_{2}(B) \frac{k}{n}+\frac{k}{2} c_{n} .
\end{gathered}
$$

Taking the limit as $n$ tends to infinity we have that

$$
\lim _{n \rightarrow \infty} \sup _{\underline{x} \in B}\left|f_{L_{n}}(\underline{x})-f_{K}(\underline{x})\right|=0 .
$$

\section{Linearization and 0-1 programming}

If $n \in \mathbb{N}$ is fixed, then we have essentially the following optimization problem in step 6

$$
\text { Minimize: } \begin{gathered}
\sum_{\underline{y}_{i j} \in G_{K}^{n}}\left(f_{L}\left(\underline{y}_{i j}\right)-f_{K}\left(\underline{y}_{i j}\right)\right) \\
\text { such that } \quad L \in \mathcal{H} .
\end{gathered}
$$

Since the coordinate X-rays of $K$ are given, the value of $f_{K}$ is given in every point. Hence

$$
\sum_{\underline{y}_{i j} \in G_{K}^{n}} f_{K}\left(\underline{y}_{i j}\right)=: C_{0}
$$

is a fixed constant and the optimization problem reduces to

$$
\text { Minimize: } \sum_{\underline{y}_{i j} \in G_{K}^{n}} f_{L}\left(\underline{y}_{i j}\right)
$$


such that $L \in \mathcal{H}$.

Let $L$ be a set in $\mathcal{H}$. Define the variables

$$
x_{k l}=\left\{\begin{array}{ll}
1 & \text { if } B_{k l}^{n} \subset L \\
0 & \text { otherwise }
\end{array} \quad(k, l=1, \ldots, n) .\right.
$$

Then

$$
f_{L}=\sum_{k, l=1}^{n} x_{k l} f_{B_{k l}^{n}}
$$

and

$$
\sum_{\underline{y}_{i j} \in G_{K}^{n}} \sum_{k, l=1}^{n} x_{k l} f_{B_{k l}^{n}}\left(\underline{y}_{i j}\right)=\sum_{k, l=1}^{n} x_{k l} \sum_{\underline{y}_{i j} \in G_{K}^{n}} f_{B_{k l}^{n}}\left(\underline{y}_{i j}\right) .
$$

Here

$$
\sum_{\underline{y}_{i j} \in G_{K}^{n}} f_{B_{k l}^{n}}\left(\underline{y}_{i j}\right)=: F_{k l}
$$

are fixed constants for every $k, l=1, \ldots, n$. Thus the optimization problem has the following form

$$
\begin{gathered}
\text { Minimize: } \sum_{k, l=1}^{n} x_{k l} F_{k l} \\
\text { such that } L=\bigcup\left\{B_{k l}^{n} \mid x_{k l}=1\right\} \in \mathcal{H} .
\end{gathered}
$$

Definition 6 A 0 -1 matrix $A=\left(a_{i j}\right)$ of size $n \times n$ is called an interval matrix if for every row $i \in\{1, \ldots, n\}$ there are indices $j_{1}, j_{2} \in\{1, \ldots, n\}$, $j_{1} \leq j_{2}$ such that

$$
a_{i j}= \begin{cases}1 & \text { if } j_{1} \leq j \leq j_{2} \\ 0 & \text { otherwise }\end{cases}
$$

Theorem 8 The compact set $L=\bigcup\left\{B_{k l}^{n} \mid x_{k l}=1\right\}$ is an element of $\mathcal{H}$ if and only if the following inequalities hold:

$$
\begin{gathered}
\sum_{i=1}^{n} x_{i 1} \geq 1 \\
\sum_{i=1}^{n} x_{i n} \geq 1 \\
\left(\prod_{j=1}^{l}\left(1-x_{k j}\right)+\prod_{j=l}^{n}\left(1-x_{k j}\right)\right)\left(\prod_{i=1}^{k}\left(1-x_{i l}\right)+\prod_{i=k}^{n}\left(1-x_{i l}\right)\right) \geq 1-x_{k l}, \\
(k, l=1, \ldots, n) \\
\sum_{j=2}^{n} x_{k j-1} x_{k+1 j}+\sum_{j=1}^{n} x_{k j} x_{k+1 j}+\sum_{j=1}^{n-1} x_{k j+1} x_{k+1 j} \geq 1
\end{gathered}
$$




$$
\begin{gathered}
(k=1, \ldots, n-1) \\
\sum_{i, j=1}^{n} x_{i j} f_{B_{i j}^{n}}\left(\underline{y}_{i j}\right) \geq f_{K}\left(\underline{y}_{i j}\right) \quad\left(\underline{y}_{i j} \in G_{K}^{n}\right) .
\end{gathered}
$$

Proof. The variables $x_{i j}(i, j=1, \ldots, n)$ form a $0-1$ matrix $A=\left(x_{i j}\right)$ of size $n \times n$. First let us assume that $L \in \mathcal{H}$. Then $B_{K}$ is the axis parallel bounding box of $L$ hence every row and column of $A$ must contain at least one element equal to 1 . The left hand sides of the inequalities (3) give the sum of the elements of the first and last column thus these inequalities must hold. Furthermore the hv-convexity of $L$ gives that $A$ and its transpose $A^{T}$ are interval matrices. Let's take an arbitrary element $x_{k l}$ of the matrix $A$. If $x_{k l}=1$ then the corresponding inequality in (4) is redundant. If $x_{k l}=0$ then all the elements $x_{k j}(j=1, \ldots, l)$ or all the elements $x_{k j}(j=l, \ldots, n)$ must be 0 since $A$ is an interval matrix. Similarly all the elements $x_{i l}(i=1, \ldots, k)$ or all the elements $x_{i l}(i=k, \ldots, n)$ must be 0 since $A^{T}$ is an interval matrix. This gives that both factors of the left hand side of (4) is at least 1 and the inequality must hold. Consider now an arbitrary row of $A$ with index $k \in\{1, \ldots, n-1\}$. By the connectedness of $L$ (Remark 2) the next row contains an element $x_{k+1 j}=1$ such that at least one of $x_{k j-1}, x_{k j}$ and $x_{k j+1}$ is 1 . This gives that at least one of the addends on the left hand sides of (5) must be 1 and these inequalities hold. Finally inequalities (6) are an immediate consequence of $f_{L}\left(\underline{y}_{i j}\right) \geq f_{K}\left(\underline{y}_{i j}\right)$ for all $\underline{y}_{i j} \in G_{K}^{n}$.

Now let us assume that the inequalities (3), (4), (5), (6) all hold. Let's look at first (4) and take an arbitrary element $x_{k l}$ of the matrix $A$. If $x_{k l}=1$ then the corresponding inequality is redundant. If $x_{k l}=0$ then the left hand side of (4) is at least 1 . This happens only if both factors on the left are at least 1 . That means at least one of the products $\prod_{j=1}^{l}\left(1-x_{k j}\right), \prod_{j=l}^{n}\left(1-x_{k j}\right)$ and one of the products $\prod_{i=1}^{k}\left(1-x_{i l}\right), \prod_{i=k}^{n}\left(1-x_{i l}\right)$ must be 1 . Such a product can only be 1 if all of its factors are 1 . This gives that the matrix $A$ and its transpose $A^{T}$ are interval matrices. On the other hand (5) ensures us that each row (except the first) must contain an element $x_{k+1 j}=1$, otherwise the left hand side would be zero. Furthermore one of the elements $x_{k j-1}, x_{k j}$ and $x_{k j+1}$ in the previous row is 1 at the same time. Thus $L$ is connected since $A$ is an interval matrix. The inequalities (5) and (3) imply that the first and the last row of $A$ and the first and the last column of $A$ must contain at least one element that is equal to 1 , hence the axis parallel bounding box of $L$ is $B_{K}$. We also have that $L$ is hv-convex because $A$ and $A^{T}$ are interval matrices and $L$ is connected. Finally inequalities (6) simply give that $f_{L}\left(\underline{y}_{i j}\right) \geq f_{K}\left(\underline{y}_{i j}\right)$ for all $\underline{y}_{i j} \in G_{K}^{n}$.

Due to Theorem 8, we can reformulate the optimization problem as follows:

$$
\text { Minimize: } \quad \sum_{k, l=1}^{n} x_{k l} F_{k l}
$$


such that $x_{k l} \in\{0,1\}(k, l=1, \ldots, n)$ and inequalities (3), (4), (5), (6) hold.

Here we have $n^{2}$ variables, $n^{2}+2$ linear and $n^{2}+n-1$ nonlinear inequalities. This is a constrained polynomial 0-1 programming problem and there are several methods to solve such a problem (see Chapters 11 and 12 in [27]). One of them is a linearization method based on the following theorem.

Theorem 9 (Li,Sun[27]) Consider the general polynomial term

$$
y=\prod_{(i, j) \in S} x_{i j}^{r_{i j}},
$$

where $x_{i j} \in\{0,1\}(i, j=1, \ldots, n), S \subset\{(i, j) \mid i, j=1, \ldots, n\}$ and $r_{i j} \in \mathbb{N}^{+}$, $r=\sum_{(i, j) \in S} r_{i j}$. Then (7) holds if and only if

$$
\begin{aligned}
& \sum_{(i, j) \in S} r_{i j} x_{i j}-y \leq r-1, \\
& -\sum_{(i, j) \in S} r_{i j} x_{i j}+r y \leq 0 .
\end{aligned}
$$

This linearization method is not effective in general because the number of the polynomial terms in (7) can be extremely large. Unfortunately this happens here if we expand the left hand side of (4). However we can handle this by defining the new variables

$$
z_{i j}:=1-x_{i j}
$$

Then inequalities (3) and (6) are

$$
\begin{gathered}
n-\sum_{i=1}^{n} z_{i 1} \geq 1 \quad n-\sum_{i=1}^{n} z_{i n} \geq 1, \\
f_{B_{K}}\left(\underline{y}_{i j}\right)-\sum_{i, j=1}^{n} z_{i j} f_{B_{i j}}\left(\underline{y}_{i j}\right) \geq f_{K}\left(\underline{y}_{i j}\right) \quad\left(\underline{y}_{i j} \in G_{K}^{n}\right),
\end{gathered}
$$

While inequalities (4) and (5) have the form

$$
\begin{gathered}
\prod_{i=1}^{k} z_{i l} \prod_{j=1}^{l} z_{k j}+\prod_{i=k}^{n} z_{i l} \prod_{j=1}^{l} z_{k j}+\prod_{i=1}^{k} z_{i l} \prod_{j=l}^{n} z_{k j}+\prod_{i=k}^{n} z_{i l} \prod_{j=l}^{n} z_{k j} \geq z_{k l}, \\
(k, l=1, \ldots, n) \\
(3 n-2)-\sum_{j=2}^{n}\left(z_{k j-1}+z_{k+1 j}\right)-\sum_{j=1}^{n}\left(z_{k j}+z_{k+1 j}\right)-\sum_{j=1}^{n-1}\left(z_{k j+1}+z_{k+1 j}\right)+ \\
\sum_{j=2}^{n} z_{k j-1} z_{k+1}+\sum_{j=1}^{n} z_{k j} z_{k+1 j}+\sum_{j=1}^{n-1} z_{k j+1} z_{k+1 j} \geq 1 .
\end{gathered}
$$




$$
(k=1, \ldots, n-1)
$$

The new objective function is

$$
\sum_{k, l=1}^{n}\left(1-z_{k l}\right) F_{k l}=\sum_{k, l=1}^{n} F_{k l}-\sum_{k, l=1}^{n} z_{k l} F_{k l} .
$$

Minimizing (12) is equivalent to maximizing

$$
\sum_{k, l=1}^{n} z_{k l} F_{k l}
$$

Rewrite (10) as follows:

$$
u_{k l}^{1}+u_{k l}^{2}+u_{k l}^{3}+u_{k l}^{4} \geq z_{k l} \quad(k, l=1, \ldots, n)
$$

where $u_{k l}^{h}$ corresponds to the h-th term, $h \in\{1, \ldots, 4\}$ of the left hand side of (10). Then, by applying Theorem 9 we get:

$$
\begin{aligned}
& \sum_{i=1}^{k} z_{i l}+\sum_{j=1}^{l} z_{k j}-u_{k l}^{1} \leq k+l-1 \quad(k, l=1, \ldots, n), \\
& \sum_{i=k}^{n} z_{i l}+\sum_{j=1}^{l} z_{k j}-u_{k l}^{2} \leq n-k+l \quad(k, l=1, \ldots, n), \\
& \sum_{i=1}^{k} z_{i l}+\sum_{j=l}^{n} z_{k j}-u_{k l}^{3} \leq n+k-l \quad(k, l=1, \ldots, n), \\
& \sum_{i=k}^{n} z_{i l}+\sum_{j=l}^{n} z_{k j}-u_{k l}^{4} \leq 2 n-k-l+1 \quad(k, l=1, \ldots, n), \\
& \quad-\sum_{i=1}^{k} z_{i l}-\sum_{j=1}^{l} z_{k j}+(k+l) u_{k l}^{1} \leq 0 \quad(k, l=1, \ldots, n), \\
& -\sum_{i=k}^{n} z_{i l}-\sum_{j=1}^{l} z_{k j}+(n-k+l+1) u_{k l}^{2} \leq 0 \quad(k, l=1, \ldots, n), \\
& -\sum_{i=1}^{k} z_{i l}-\sum_{j=l}^{n} z_{k j}+(n+k-l+1) u_{k l}^{3} \leq 0 \quad(k, l=1, \ldots, n), \\
& -\sum_{i=k}^{n} z_{i l}-\sum_{j=l}^{n} z_{k j}+(2 n-k-l+2) u_{k l}^{4} \leq 0 \quad(k, l=1, \ldots, n) .
\end{aligned}
$$


Denoting $z_{k j-1} z_{k+1 j}=v_{k j}^{1}, z_{k j} z_{k+1 j}=v_{k j}^{2}$, and $z_{k j+1} z_{k+1 j}=v_{k j}^{3}$, we can rewrite (11) as follows:

$$
\begin{gathered}
(3 n-2)-\sum_{j=2}^{n}\left(z_{k j-1}+z_{k+1}\right)-\sum_{j=1}^{n}\left(z_{k j}+z_{k+1 j}\right)-\sum_{j=1}^{n-1}\left(z_{k j+1}+z_{k+1}\right)+ \\
\sum_{j=2}^{n} v_{k j}^{1}+\sum_{j=1}^{n} v_{k j}^{2}+\sum_{j=1}^{n-1} v_{k j}^{3} \geq 1 . \quad \text { (16) } \\
(k=1, \ldots, n-1)
\end{gathered}
$$

Then, by applying Theorem 9, we get

$$
\begin{aligned}
z_{k j-1}+z_{k+1 j}-v_{k j}^{1} \leq 1 \quad(j, k=1, \ldots, n ; j \neq 1, k \neq n), \\
z_{k j}+z_{k+1 j}-v_{k j}^{2} \leq 1 \quad(j, k=1, \ldots, n ; k \neq n), \\
z_{k j+1}+z_{k+1 j}-v_{k j}^{3} \leq 1 \quad(j, k=1, \ldots, n-1), \\
-z_{k j-1}-z_{k+1 j}+2 v_{k j}^{1} \leq 0 \quad(j, k=1, \ldots, n ; j \neq 1, k \neq n), \\
-z_{k j}-z_{k+1 j}+2 v_{k j}^{2} \leq 0 \quad(j, k=1, \ldots, n ; k \neq n), \\
-z_{k j+1}-z_{k+1 j}+2 v_{k j}^{3} \leq 0 \quad(j, k=1, \ldots, n-1) .
\end{aligned}
$$

Theorem 10 The nonlinear problem in step 6 of the reconstruction algorithm is equivalent to the following linear integer programming problem.

$$
\text { Maximize: } \quad \sum_{k, l=1}^{n} z_{k l} F_{k l}
$$

such that

$$
\begin{aligned}
& z_{k l} \in\{0,1\}(k, l=1, \ldots, n), \\
& u_{k l}^{i} \in\{0,1\}(k, l=1, \ldots, n),(i=1, \ldots, 4), \\
& v_{k, j}^{1} \in\{0,1\}(j, k=1, \ldots, n ; j \neq 1, k \neq n), \\
& v_{k, j}^{2} \in\{0,1\}(j, k=1, \ldots, n ; k \neq n), \\
& v_{k, j}^{3} \in\{0,1\} \quad(j, k=1, \ldots, n-1),
\end{aligned}
$$

and inequalities (8), (9), (13), (14), (15), (16), (17), (18) hold.

Here we have $n^{2}+4 n^{2}+(n-1)(3 n-2)=8 n^{2}-5 n+2$ variables and $2+n^{2}+n^{2}+8 n^{2}+n-1+2(n-1)(3 n-2)=16 n^{2}-9 n+5$ linear inequalities. There is a wide literature on linear $0-1$ programming problems and we can take any method to solve this one. 

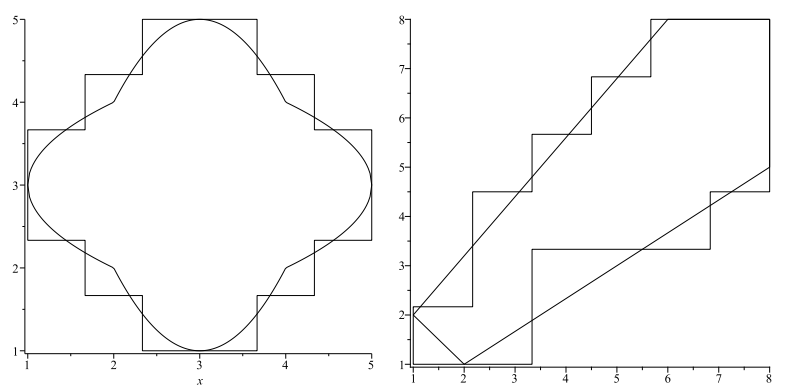

Figure 1: Outputs of the algorithm, $\mathrm{n}=6$
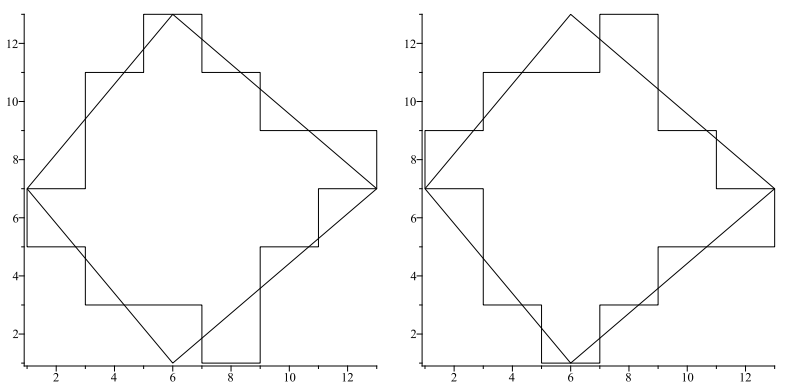

Figure 2: Different optimal solutions

In Figure 1 we can see two of the outputs of the algorithm with resolution $n=6$. On the left the input data is the coordinate X-ray functions of the set

$$
D_{1}=\left\{\left(x_{1}, x_{2}\right) \in \mathbb{R}^{2} \mid 1 \leq x_{1} \leq 5 \text { and } f\left(x_{1}\right) \leq x_{2} \leq g\left(x_{1}\right)\right\},
$$

where

$$
f\left(x_{1}\right)=\left\{\begin{aligned}
-\sqrt{x_{1}-1}+3 & \text { if } 1 \leq x_{1}<2 \\
\left(x_{1}-3\right)^{2}+1 & \text { if } 2 \leq x_{1}<4 \\
-\sqrt{-x_{1}+5}+3 & \text { if } 4 \leq x_{1} \leq 5 \\
0 & \text { otherwise }
\end{aligned}\right.
$$

and

$$
g\left(x_{1}\right)=\left\{\begin{aligned}
\sqrt{x_{1}-1}+3 & \text { if } 1 \leq x_{1}<2 \\
-\left(x_{1}-3\right)^{2}+5 & \text { if } 2 \leq x_{1}<4 \\
\sqrt{-x_{1}+5}+3 & \text { if } 4 \leq x_{1} \leq 5 \\
0 & \text { otherwise. }
\end{aligned}\right.
$$

On the right the input data is the coordinate X-ray functions of the set

$$
D_{2}=\operatorname{conv}\{(2,1) ;(8,5) ;(8,8) ;(6,8) ;(1,2)\} \text {. }
$$

It is possible that there are more than one minimizing set in $\mathcal{H}$ in step 6 or step $6^{*}$ of the algorithm even if the coordinate $\mathrm{X}$-rays uniquely determine the 
corresponding set. For example the algorithm returns the only optimal solution for $D_{1}$ and $D_{2}$, while Figure 2 shows two of the four optimal solutions for the tetragon

$$
D_{3}=\operatorname{conv}\{(1,7) ;(6,1) ;(13,7) ;(6,13)\} \text {. }
$$

If we would like to find all solutions we can do the following. Let $L$ be one solution, let $\left(\widehat{z}_{i j}\right)$ be the matrix that represent $L$ and let

$$
S=\left\{(i, j) \in \mathbb{N} \times \mathbb{N} \mid \widehat{z}_{i j}=1,1 \leq i, j \leq n\right\} .
$$

Then the linear inequality

$$
\sum_{(i, j) \in S} z_{i j}-\sum_{\substack{1 \leq i, j \leq n \\(i, j) \notin S}} z_{i j} \leq|S|-1
$$

is not satisfied by the variables $\widehat{z}_{i j}(i, j=1, \ldots, n)$, but if we change any of them, then (20) will be satisfied. This means that if we add the inequality (20) to the system of inequalities of problem (19), then $L$ won't be a solution anymore, but no other feasible point will be excluded. We can solve the new linear programming problem and obtain a solution $L^{*}$. If the value of the objective function is the same as for the optimal solution of the problem (19), then $L^{*}$ is another optimal solution for the problem (19). After that we can exclude $L^{*}$ from the set of feasible points with the same technique as above. We continue this procedure until the value of the objective function changes. Then we get all the optimal solutions of (19).

\section{$7 \quad$ Greedy algorithm and voting}

The algorithm for reconstruction provided in the previous section has a clear proof of convergence, but integer programming is NP-hard and it makes this algorithm quite slow. We can obtain a faster algorithm by using a greedy algorithm, although the proof of convergence does not work for that case.

\section{The greedy algorithm for reconstruction}

Input: $n \in \mathbb{N}$ and $X_{1} K, X_{2} K$, the coordinate X-rays of a connected hvconvex body $K \subset \mathbb{R}^{2}$ with $K=\operatorname{cl}(\operatorname{int}(K))$ and having axis parallel bounding box $B_{K}$.

STEP 1-4: The same steps as in the algorithm of section 4 .

The following steps are based on finding the subrectangle $D$ that causes the greatest amount of decreasing of the difference of the conic functions. STEP 5: First let $L_{n}$ be the union of all subrectangles, i.e. $L_{n}=B_{K}$. 

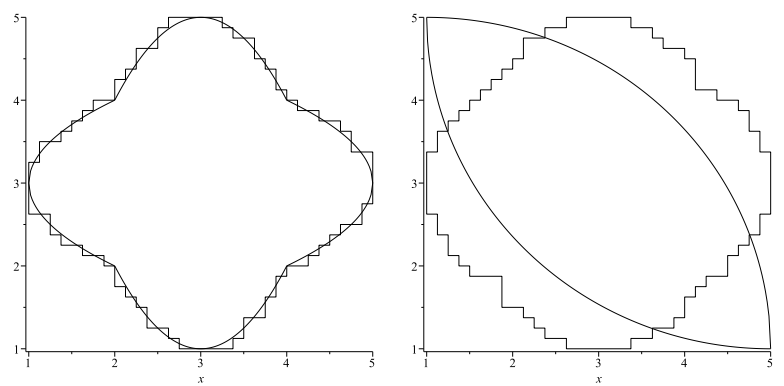

Figure 3: Outputs of the greedy algorithm

STEP 6: Let $\mathcal{D}$ be the collection of all subrectangles $D$ such that $L_{n} \backslash D \in \mathcal{H}$ (where $\mathcal{H}$ is defined in step 5 of the algorithm in section 4 ).

STEP 7: If $\mathcal{D} \neq \emptyset$ then choose $D$ from $\mathcal{D}$ that minimizes

$$
\max _{\underline{y}_{i j} \in G_{K}^{n}}\left(f_{L_{n} \backslash D}\left(\underline{y}_{i j}\right)-f_{K}\left(\underline{y}_{i j}\right)\right) .
$$

Then delete $D$ from $L_{n}$ and continue with step 6 . If $\mathcal{D}=\emptyset$ the algorithm ends.

\section{Output: $L_{n}$}

In Figure 3 two of the outputs of the greedy algorithm are presented for coordinate X-ray functions of the sets $D_{1}$ and

$$
D_{4}=\left\{\left(x_{1}, x_{2}\right) \in \mathbb{R}^{2} \mid 1 \leq x_{1} \leq 5 \text { and } f_{2}\left(x_{1}\right) \leq x_{2} \leq g_{2}\left(x_{1}\right)\right\},
$$

where

$$
f_{2}\left(x_{1}\right)=5-\sqrt{16-\left(x_{1}-5\right)^{2}} \quad \text { and } \quad g_{2}\left(x_{1}\right)=1+\sqrt{16-\left(x_{1}-1\right)^{2}} .
$$

We can see that the output is very close to $D_{1}$ on the left, however it is quite far from $D_{4}$ on the right with respect to the Hausdorff distance. This is because the subrectangles in the corners tend to add more to the value of $f_{L_{n}}$ in the vertices of the control grid than the subrectangles in the middle. So the subrectangles in the corners are the first candidates to be deleted in step 6 . Thus the greedy algorithm is not suitable for sets that reach some corners of their bounding box.

On the other hand we also note that in step 7 of the greedy algorithm it is possible that there are more than one minimizing subrectangles. In these cases we need to decide how to choose one of them. One evident decision is to choose one randomly. This way we obtain a fast stochastic algorithm, which allows us to use the following voting procedure.

Let $k \in \mathbb{N}$ be fixed and run the stochastic greedy algorithm $k$-times. We say that an output votes for a subrectangle if the output contains this 

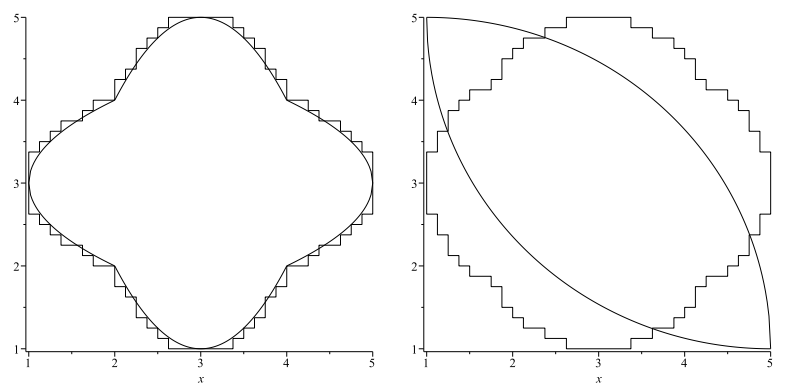

Figure 4: Voting with the greedy algorithm

subrectangle. Then we choose a real number $q \in[0,1]$ called the quota and let $L_{n}$ be the union of all those subrectangles having at least $k \cdot q$ votes. The extreme cases are $q=1$ and $q \leq 1 / k$ when we take the intersection and the union of all the outputs respectively. The intersection of outputs shows us the subrectangles that are very likely to be in the optimal solution, however the subrectangles that are not in the union of outputs are very likely to be excluded by the optimal solution. We can note that the intersection of the outputs is also hv-convex but it may have a smaller bounding box than the original outputs. On the other hand the union of the outputs has the same bounding box as the original outputs, but it is not necessarily hv-convex. These problems can be reduced if we sort the outputs by cluster analysis using the Hausdorff distance. In Figure 4 we can see two outputs of the voting procedure with $k=10$ and $q=1 / 10$. In the case of $D_{1}$ the approximation is better than ever before, but in the case of $D_{4}$ the voting procedure doesn't help us to get closer to the original set. This is because the subrectangles in the corners add so much to the value of $f_{L_{n}}$ in the vertices of the control grid that they are deleted in the first steps every time the greedy algorithm runs. To avoid this problem we need a different way of thinking.

\section{The anti-greedy algorithm}

In the previous section we discussed how the subrectangles in the corners cause a problem during the greedy algorithm. Furthermore we observe that the result of the voting procedure for $D_{4}$ in Figure 4 is very similar to the single output of the greedy algorithm in Figure 3. This means that every time the greedy algorithm runs the same subrectangles are deleted and the outputs slightly differ from each other. The greedy algorithm starts to delete those subrectangles that add the most to the value of $f_{L_{n}}$ in the control points so this value starts to decrease fast. But there's a lower bound for this value, namely the value of $f_{K}$. Thus the algorithm reaches a point soon when no more subrectangles can be deleted due to the lower bound. Now it's an evident idea to delete those subrectangles that add little to the value 

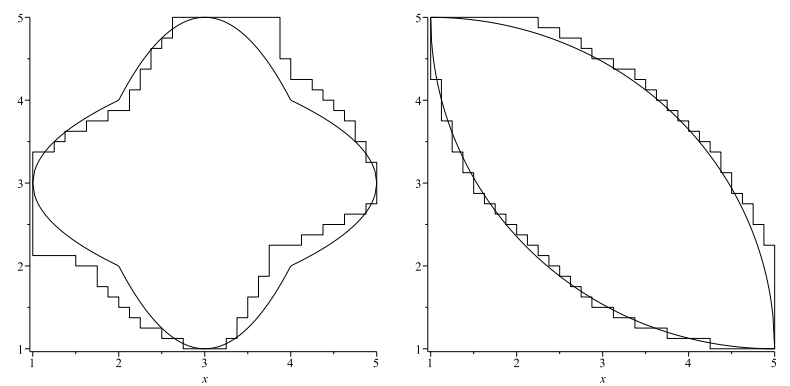

Figure 5: Voting with the anti-greedy algorithm

of $f_{L_{n}}$ in the control points. This way we can choose from a larger set of subrectangles after deleting one than before because the values of $f_{L_{n}}$ in the control points don't get close to the lower bound early. This gives us more variability of the outputs and the voting procedure can be more effective. However if we make these changes the algorithm cannot be called greedy but anti-greedy. So we have now an anti-greedy algorithm for reconstruction that has the same steps as the greedy algorithm except step 7 that is changed to choose $D$ from $\mathcal{D}$ that maximizes

$$
\max _{\underline{y}_{i j} \in G_{K}^{n}}\left(f_{L_{n} \backslash D}\left(\underline{y}_{i j}\right)-f_{K}\left(\underline{y}_{i j}\right)\right) .
$$

Figure 5 shows two outputs of the anti-greedy algorithm. We can see that the output for $D_{4}$ on the right is much better than before, but it gets a little worse for $D_{1}$ on the right. This happens because the anti-greedy algorithm tends to keep the subrectangles in the corners. So there are sets that can be approximated better with the greedy algorithm and there are other sets that are better to be approximated with the anti-greedy algorithm. It is an interesting question how to choose in advance if the greedy or the anti-greedy algorithm must be performed. We might need additional data in more directions.

\section{Acknowledgements}

Ábris Nagy has been supported, in part, by the Hungarian Academy of Sciences, the European Union and the State of Hungary, co-financed by the European Social Fund in the framework of TÁMOP 4.2.4. A/2-11-1-20120001 'National Excellence Program'.

Csaba Vincze was partially supported by the European Union and the European Social Fund through the project Supercomputer, the national virtual lab (grant no.:TÁMOP-4.2.2.C-11/1/KONV-2012-0010). This work is supported by the University of Debrecen's internal research project. 


\section{References}

[1] A. Alpers, R. J. Gardner, S. Knig, R. S. Pennington, C. B. Boothroyd, L. Houben, R. E. Dunin-Borkowski and K. J. Batenburg. Geometric reconstruction methods for electron tomography. Ultramicroscopy, 128 (2013) 42-54.

[2] P Balázs. A benchmark set for the reconstruction of hv-convex discrete sets. Discrete Applied Mathematics, 157 (2009) 3447-3456.

[3] E. Balogh, A. Kuba, C. Dévényi A. Del Lungo and R. Pinzani. Comparison of algorithms for reconstructing hv-convex discrete sets. Linear Algebra and its Applications, 339 (2001) 23-35.

[4] E. Barcucci, A. Del Lungo, M. Nivat and R. Pinzani. Reconstructing convex polyominoes from horizontal and vertical projections. Theoretical Computer Science, 155 (1996) 321-347.

[5] K. J. Batenburg and J. Sijbers. DART: a practical reconstruction algorithm for discrete tomography. IEEE Transactions on Image Processing, (9) 20 (2011) 2542-2553.

[6] S. Brunetti and A. Daurat. An algorithm reconstructing lattice convex sets. Theoretical Computer Science, 304 (2003) 35-57.

[7] S. Brunetti and A. Daurat. Determination of Q-convex bodies by Xrays. Electronic Notes in Discrete Mathematics, 20 (2005) 67-81.

[8] S. Brunetti and A. Daurat. Stability in discrete tomography: Some positive results. Discrete and Applied Mathematics, 147 (2005) 207226.

[9] S. Brunetti, P. Dulio and C. Peri. Discrete tomography determination of bounded lattice sets from four X-rays. Discrete Applied Mathematics, 161 (15) (2013) 2281-2292.

[10] P. Dulio. Convex decomposition of U-polygons. Theoretical Computer Science, 406/1-2 (2008) 80-89.

[11] P. Dulio, R. J. Gardner and C. Peri. Discrete point X-rays. SIAM Journal of Discrete Mathematics, 20(1) (2006) 171-188.

[12] P. Dulio and C. Peri. On the geometric structure of lattice U-polygons. Discrete Mathematics, 307/19-20 (2007) 2330-2340.

[13] P. C. Fishburn and L. A. Shepp. Sets of uniqueness and additivity in integer lattices. in Discrete Tomography: Foundations, Algorithms and Applications, ed. by G. T. Herman and A. Kuba, Birkhäuser, Boston, (1999) 35-58. 
[14] R. J. Gardner. Geometric Tomography. Cambridge University Press, New-York, 1995, second ed., 2006.

[15] R. J. Gardner and P. Gritzmann. Discrete tomography: Determination of finite sets by X-rays. Transactions of the American Mathematical Society, 349 (1997) 2271-2295.

[16] R. J. Gardner and P. Gritzmann. Uniqueness and complexity in discrete tomography. in Discrete Tomography: Foundations, Algorithms and Applications, ed. by G. T. Herman and A. Kuba, Birkhäuser, Boston, (1999) 85-113.

[17] R. J. Gardner and M. Kiderlen. A solution to Hammer's X-ray reconstruction problem. Advances in Mathematics, 214 (2007) 323-343.

[18] R. J. Gardner and P. McMullen. On Hammer's X-ray problem. Journal of the London Mathematical Society, (2) 21 (1980) 171-175.

[19] P. Gritzmann, B. Langfeld and M. Wiegelmann. Uniqueness in discrete tomography: three remarks and a corollary. SIAM Journal of Discrete Mathematics, 25 (2011) 1589-1599.

[20] L. Hajdu. Unique reconstruction of bounded sets in discrete tomography. Electronic Notes in Discrete Mathematics, 20 (2005) 15-25.

[21] M. A Haque, M. O. Ahmad, M. N. S. Swamy, M. K. Hasan and S. Y. Lee. Adaptive projection selection for computed tomography. IEEE Transactions on Image Processing, 22(12) (2013) 5085-5095.

[22] G. T. Herman. Reconstruction of binary patterns from a few projections, in: A. Günther, B. Levrat, H. Lipps (Eds.), International Computing Symposium 1973. North-Holland Publ. Co., Amsterdam, (1974) $371-378$.

[23] G. T. Herman. Fundamentals of Computerized Tomography: Image Reconstruction from Projections. Springer, London, 2009.

[24] C. Huck and M. Spieß. Solution of a uniqueness problem in the discrete tomography of algebraic Delone sets. Journal für die reine und angewandte Mathematik, 677 (2013) 199-224.

[25] A. C. Kak and M. Slaney. Principles of Computerized Tomographic Imaging. Society of Industrial and Applied Mathematics, Philadelphia, PA, 2001.

[26] D. Kölzow, A. Kuba and A. Volčič. An algorithm for reconstructing convex bodies from their projections. Discrete and Computational Geometry, 4 (1989) 205-237. 
[27] D. Li and X. Sun. Nonlinear Integer Programming. Springer, New-York, 2006.

[28] Cs. Vincze and Á. Nagy. On the theory of generalized conics with applications in geometric tomography. Journal of Approximation Theory, 164 (2012) 371-390.

[29] Cs. Vincze and Á. Nagy. Generalized conic functions of hv-convex planar sets: continuity properties and relations to X-rays. Submitted to Aequationes Mathematicae, arXiv:1303.4412.

[30] A. Volčič. A three-point solution to Hammer's X-ray problem. Journal of the London Mathematical Society, 34 (1986) 349-359.

[31] Z. Yu, J. B. Thibault, C. A. Bouman, K. D. Sauer and J. Hsieh. Fast model-based X-ray CT reconstruction using spatially nonhomogeneous ICD optimization. IEEE Transactions on Image Processing, 20(1) (2011) 161-175.

[32] J. Zhang, J. Wang, H. Zuo, G. Xu, and J. B. Thibault. Compressed sensing algorithms for fan-beam computed tomography image reconstruction. Optical Engineering, 51(7) (2012) 071402. 